\title{
Laparoscopic Roux-en-Y gastric bypass or vertical sleeve gastrectomy: a weighty decision
}

\author{
Emma J. Osland ${ }^{1,2}$, Muhammed Ashraf Memon, ${ }^{3,4,5,6,7}$ \\ ${ }^{1}$ Department of Nutrition and Dietetics, Royal Brisbane and Women's Hospital, Brisbane, Australia; ${ }^{2}$ Department of Human Movement and \\ Nutrition, University of Queensland, Brisbane, Australia; ${ }^{3}$ School of Agricultural, Computational and Environmental Sciences, International Centre \\ for Applied Climate Sciences and Centre for Health Sciences Research, University of Southern Queensland, Toowoomba, Australia; ${ }^{4}$ Sunnybank \\ Obesity Centre and South East Queensland Surgery (SEQS) Brisbane, Australia; ${ }^{5}$ Mayne Medical School, School of Medicine, University of \\ Queensland, Brisbane, Australia; ${ }^{6}$ Faculty of Health Sciences and Medicine, Bond University, Gold Coast, Australia; ${ }^{7}$ Faculty of Health and Social \\ Science, Bolton University, Bolton, UK \\ Correspondence to: Professor Muhammed Ashraf Memon. Sunnybank Obesity Centre and South East Queensland Surgery, Suite 9, McCullough \\ Centre, 259 McCullough Street, Sunnybank, Qld 4109, Australia. Email: mmemon@yahoo.com. \\ Provenance: This is an invited Editorial commissioned by the Section Editor Kaiping Zhang (AME College, AME Group, China). \\ Comment on: Peterli R, Wölnerhanssen BK, Peters T, et al. Effect of Laparoscopic Sleeve Gastrectomy vs Laparoscopic Roux-en-Y Gastric Bypass on \\ Weight Loss in Patients With Morbid Obesity: The SM-BOSS Randomized Clinical Trial. JAMA 2018;319:255-65.
}

Submitted Oct 12, 2018. Accepted for publication Oct 15, 2018.

doi: $10.21037 /$ atm.2018.10.43

View this article at: http://dx.doi.org/10.21037/atm.2018.10.43

The prevalence of obesity has tripled internationally in the last four decades, affecting an estimated 650 million of the world's population (1). Obesity is well recognised as an independent risk factor for the development of chronic conditions such as cardiovascular disease and diabetes, as well as some forms of cancer (1). Thus, efficacious and costeffective methods of managing obesity and its associated comorbidities are required. Since the 1990s bariatric surgical procedures have become increasingly common as a surgical option for dealing with morbid obesity (2). In this context, the laparoscopic Roux-en-Y gastric bypass (LRYGB) has been considered the gold standard with its established longterm outcomes. However, the newer laparoscopic vertical sleeve gastrectomy (LVSG) procedure is rapidly overtaking LRYGB because of its simplicity, short learning curve and rapid initial weight loss (3). Nevertheless, there remains a paucity of long-term outcomes for the LVSG. Only four randomised controlled trials (RCTs) comparing five-year outcomes of the two procedures have been published to date, with the Swiss Multicentre Bypass or Sleeve Study (SMBOSS) being amongst the most recent (4-7).

SM-BOSS, published by Peterli and colleagues (4), randomized 217 morbidly obese patients between 2007 and 2011 to receive LVSG or LRYGB, investigating weight loss outcomes [described as \% of excess body mass index (BMI) loss] as their primary outcome. Weight loss was successfully achieved in both intervention groups of SM-BOSS, and despite a slow trend in weight recidivism over the reporting period, five year \% excess BMI loss from baseline was reported at $61.1 \%$ in LVSG and $68.3 \%$ LRYGB (4). Initial $\mathrm{BMI}$ was the only factor to be significantly associated with $\%$ excess BMI loss without multifactor adjustment, and a linear decrease in excess BMI was shown throughout the follow up period for both procedures (4). Post hoc analyses (comparison between baseline and five years) for the percentage of patients with $\%$ excessive BMI loss of $>50 \%$ and $>75 \%$ showed similar trends for both procedures (4). Lastly mean BMI reduction and mean weight reduction was not significantly different for both groups at 5 years (4). Contrary to the SM-BOSS findings, the three other RCTs $(5-7)$ that have investigated this topic have suggested greater weight loss at five years in LRYGB compared to LVSG. However, these differences may be either due to underpowered studies or secondary to a large number of drop outs at five years interval which may dilute the true difference between the two groups.

Secondary outcomes considered by SM-BOSS (4) included changes in comorbidities (specifically type 2 
diabetes, hypertension, sleep apnoea, back/joint pain, hyperuricemia, depression), quality of life and surgical outcomes [duration and cost of operation, and requirement of secondary intervention (revisional surgery)]. Consistent with the findings of the other RCTs reporting comorbidity outcomes at five years $(5,7)$, SM-MOSS (4) demonstrated the sustained benefit of both LRYGB and LVSG on the management of common comorbid conditions. For a number of comorbidities, there appears to be no benefit of one procedure over the other.

Gastroesophageal reflux disease (GERD) has become a hotly debated topic with the increasing utilization of LVSG as it may lead to the development of de novo GERD or aggravate a pre-existing GERD. SM-BOSS (4) demonstrated a significantly higher percentage of patients with worsening of GERD symptoms in the LVSG intervention group (i.e., 32\% vs. LRYGB cohort 6.3\%). Furthermore, after 5 years, de novo GERD developed in 32\% of LVSG patients compared to $11 \%$ in LRYGB patients (4). Ultimately $\sim 9 \%(n=9)$ of those receiving LVSG required conversion to RYGB to manage GERD symptoms during the five year follow up period, while medical management was sufficient to manage GERD in the LRYGB recipients (4). Revisional surgery for the management of GERD has been reported in two other RCTs $(5,6)$. SLEEVEPASS $(5)$ reports GERD as both major and minor late complications in their LVSG patients, requiring conversion to LRYGB in $5.8 \%$ of patients and medication management in a further $9.1 \%$. Similarly, Ignat et al. (6) report two LVSG conversions to RYGB for severe GERD, with additional patients requiring medical management. However, the interpretation of these outcomes is confounded by the lack of description regarding the presence or absence of hiatal hernia in patients receiving surgery. Similarly, concomitant hiatal hernia repair within procedures and the preoperative use of physiological testing, such as 24-hour $\mathrm{pH}$ study and high-resolution manometry, is not consistently reported. It is unfortunate that the utilization of preoperative $\mathrm{pH}$ study and manometry have not yet been adopted as standard practice in bariatric patients. Their widespread use, therefore would not only provide the esophageal pressure topography and 24-hour $\mathrm{pH}$ profile, but would assist in the selection of the bariatric procedure best suited to an individual's GERD risk profile. This approach may in turn reduce the need for surgical revisions in this patient population, as well as the potential for postoperative GERD related complications such as Barrett's esophagus. With an increasing number of case reports describing the development of esophageal neoplasms following LVSG $(8,9)$, the long-term impacts of postoperative GERD in this population require further surveillance and research.

A trend in the five-year follow-up toward remission of dyslipidaemia was observed in the LRYGB group in SMBOSS (4), ( $\mathrm{P}=0.03$ unadjusted, $\mathrm{P}=0.09$ adjusted), despite significantly more patients in the LVSG group having dyslipidemia at baseline. Similarly, the Zhang et al. (7) and SLEEVEPASS (5) studies report a higher percentage of patients in the LRGYB group who achieved remission or improvement in dyslipidaemia. The SLEEVEPASS (5) results indicated that low-density lipoprotein-cholesterol (LDL-C) was significantly lower at five years following LRYGB versus LVSG, irrespective of the presence of pre-existing dyslipidemia. However, longer term followup is required to determine whether these biochemical improvements in LDL-C translate into clinical reductions in cardiovascular disease and coronary artery disease.

LRYGB has traditionally been considered superior to LVSG for facilitating improvements in diabetes management (10), however the long-term results from SMBOSS (4) have demonstrated comparable postoperative outcomes for these two procedures. At five years postoperatively, complete type 2 diabetes remission was seen in $61.5 \%$ in the LVSG group vs. $67.9 \%$ in the LRYGB (4). Marked improvement of glycemic control was seen after five years compared with baseline, with no significant differences between the treatment groups in fasting glucose or hemoglobin A1c (4). SLEEVEPASS (5) and Zhang et al. (7) similarly showed equivalence of LVSG and LRYGB for diabetes management. However, this should be considered in the context that all studies are underpowered for secondary analysis such as diabetes outcomes.

Hypertension was reported at baseline in two thirds of the SM-BOSS (4) cohort. Remission was achieved in a significant number of patients in both groups; $62.5 \%$ in LVSG and $70.3 \%$ in LRYGB at 5 years (4). Zhang et al. (7) reports similar outcomes for hypertension as SMBOSS (4). SLEEVEPASS (5), on the other hand, reported significantly greater improvement in hypertension with LRYGB versus LVSG at 5 years, evidenced by a $51 \%$ vs. $29 \%$ discontinuation of antihypertensive medication with LRYGB $v s$. LVSG group respectively $(\mathrm{P}=0.02)$.

Late complications (defined as developing between one month to five years postoperatively) requiring further surgical or endoscopic intervention were reported in both LRYGB (17.3\%) and LVSG (15\%) groups in the SM-BOSS 
study (4). These differences were not statistically significant in uncorrected analyses (4). There was no difference between groups for requirements of revisional surgeries $(n=16$ in LVSG vs. $\mathrm{n}=23$ in LRYGB), however the reasons for these differed (GERD, incisional hernias, severe dumping, pouch revision and insufficient weight loss). No deaths were attributed directly to any of the bariatric procedures (4).

Finally, SM-BOSS (4) reported a sustained improvement in quality of life at five years following both LRYGB and LVSG, with no significant differences being identified between procedures. Despite differences in quality of life tools used, this finding is consistent with the other RCTs investigating this outcome (5-7). Observing the data, these changes in quality of life appear to roughly follow weight loss (or weight regain).

While SM-BOSS (4) and the other RCTs comparing long-term clinical outcomes for LRYGB and LVSG (5-7) add considerably to our understanding of natural history of these commonly utilised bariatric procedures, there remain several noteworthy gaps within the current data that require further consideration.

Weight loss is considered to be the primary indicator of success of a bariatric procedure, and thus features as the primary outcome for the all the RCTs discussed here. Patterns of weight change over time following bariatric procedures are incompletely understood, and long-term studies are well placed to expand our understanding of the trajectory of postoperative weight change. However, to date many of the long-term studies report data on as baseline to five year follow up, thus missing the opportunity to describe this important aspect of the postoperative course. Those that do present this data in some fashion appear to support previously reported patterns, where weight regain begins one to two years postoperatively, which to varying degrees continues throughout the observation period. The final data point, however as expected, generally remains significantly lower than at baseline. The management of weight recidivism is important and likely multifactorial (11). None of the RCTs following patients to five years detail the specific nature or intervals of the clinical follow up outside of the data collection requirements, thus the context in which weight regain is occurring remains unknown. Multidisciplinary models have been proposed to manage the complex interplay of nutritional, lifestyle, psychological, medical and surgical issues that contribute to the ongoing success of bariatric surgery (11). The importance of ongoing support for the challenges that lead to the patient requiring bariatric surgery in the first place cannot be underestimated.
Another major gap left largely unaddressed by the discussed studies is the comparative impact on micronutrient status and supplementation requirements, which, considering the anatomic and nutritional changes following both LVSG and LRYGB, should be considered a major limitation in our understanding of postoperative bariatric surgical outcomes. Despite long-term monitoring and replacement of micronutrients being recommended following bariatric surgery (12), this is an aspect of management that has been poorly described in the postoperative course (13). Given the limited reporting on long-term micronutrient outcomes, trends in micronutrient abnormalities may remain undescribed and largely unrecognised in practice.

Another consideration of long-term RCTs is the dropout rate from follow up at 5 years. SM-BOSS (4) reports the lowest with $8 \%$ loss to follow up from the primary outcome measure, however in the other studies (5-7) these range from a loss of $16 \%$ to $27 \%$ of those who originally received surgery. Studies investigating this have suggested that those lost to follow up have greater rates of weight regain post-surgery (14) and presentation with medical complications (15). Given the lack of intention to treat analyses in the largest of the RCTs investigating the outcomes between LRYGB and LVSG, this has significant implications for the interpretation of the data, as well as the translation of research outcomes into clinical practice.

SM-BOSS (4) is among the first of the RCTs with longterm follow up data to call into question the traditionally held belief that LRYGB provides superior weight loss and in particular diabetes resolution outcomes compared to LVSG. While promising outcomes were noted in terms of dyslipidemia management in the LRYGB group, longerterm surveillance is required to determine to what degree this translates into clinical outcomes such as the incidence of cardiovascular disease. Perhaps the most significant aspect of SM-BOSS is that it represents the first RCT to comprehensively describe GERD postoperative outcomes, and clearly illustrates the propensity of the LVSG to worsen existing GERD symptoms or lead to its development. The full significance of this in terms of esophageal cancer risk in this patient group warrants further investigation. While we wait further long-term outcome data to become available, an immediate application for practice should be a thorough preoperative patient assessment that incorporates physiological testing, such as high-resolution manometry and $\mathrm{pH}$ study, in the consideration of the GERD risk posed by the surgical options available. 


\section{Acknowledgements}

None.

\section{Footnote}

Conflicts of Interest: The authors have no conflicts of interest to declare.

\section{References}

1. World Health Organisation. Obesity and overweight. 2018. Available online: http://www.who.int/news-room/ fact-sheets/detail/obesity-and-overweight

2. Faria GR. A brief history of bariatric surgery. Porto Biomed J 2017;2:90-2.

3. Ozsoy Z, Demir E. Which Bariatric Procedure Is the Most Popular in the World? A Bibliometric Comparison. Obes Surg 2018;28:2339-52.

4. Peterli R, Wölnerhanssen BK, Peters T, et al. Effect of Laparoscopic Sleeve Gastrectomy vs Laparoscopic Rouxen-Y Gastric Bypass on Weight Loss in Patients With Morbid Obesity: The SM-BOSS Randomized Clinical Trial. JAMA 2018;319:255-65.

5. Salminen P, Helmiö M, Ovaska J, et al. Effect of Laparoscopic Sleeve Gastrectomy vs Laparoscopic Rouxen-Y Gastric Bypass on Weight Loss at 5 Years Among Patients With Morbid Obesity: The SLEEVEPASS Randomized Clinical Trial. JAMA 2018;319:241-54.

6. Ignat M, Vix M, Imad I, et al. Randomized trial of Rouxen- $Y$ gastric bypass versus sleeve gastrectomy in achieving excess weight loss. Br J Surg 2017;104:248-56.

Cite this article as: Osland EJ, Memon MA. Laparoscopic Roux-en-Y gastric bypass or vertical sleeve gastrectomy: a weighty decision. Ann Transl Med 2018;6(Suppl 1):S69. doi: 10.21037/atm.2018.10.43
7. Zhang $\mathrm{Y}, \mathrm{Zhao} \mathrm{H}$, Cao Z, et al. A randomized clinical trial of laparoscopic Roux-en-Y gastric bypass and sleeve gastrectomy for the treatment of morbid obesity in China: a 5-year outcome. Obes Surg 2014;24:1617-24.

8. Wright FG, Duro A, Medici JR, et al. Esophageal adenocarcinoma five years after laparoscopic sleeve gastrectomy. A case report. Int J Surg Case Rep 2017;32:47-50.

9. Masrur M, Elli E, Gonzalez-Ciccarelli LF, et al. De novo gastric adenocarcinoma 1 year after sleeve gastrectomy in a transplant patient. Int J Surg Case Rep 2016;20:10-3 .

10. Keidar A. Bariatric surgery for type 2 diabetes reversal: the risks. Diabetes Care 2011;34 Suppl 2:S361-266.

11. Karmali S, Brar B, Shi X, et al. Weight recidivism post-bariatric surgery: a systematic review. Obes Surg 2013;23:1922-33.

12. Mechanick JI, Youdim A, Jones DB, et al. Clinical practice guidelines for the perioperative nutritional, metabolic, and nonsurgical support of the bariatric surgery patient--2013 update: cosponsored by American Association of Clinical Endocrinologists, the Obesity Society, and American Society for Metabolic \&amp; Bariatric Surgery. Surg Obes Relat Dis 2013;9:159-91.

13. Lewis CA, de Jersey S, Hopkins G, et al. Does Bariatric Surgery Cause Vitamin A, B1, C or E Deficiency? A Systematic Review. Obes Surg 2018;28:3640-57.

14. Harper J, Madan AK, Ternovits CA, et al. What happens to patients who do not follow-up after bariatric surgery? Am Surg 2007;73:181-4.

15. Robson A, Brzezinska M, Shearer C. Managing bariatric surgical patients lost to follow up in other centres. Int J Surg 2017;12:S99. 